\title{
AVALIAÇÃO DOS PACIENTES PORTADORES DE LESÃO POR PRESSÃO INTERNADOS EM HOSPITAL GERAL
}

\author{
Adriana Moro*, Alice Maurici, Juliana Barros do Valle, Viviane Renata Zaclikevis, Harry Kleinubing Junior \\ Trabalho realizado no Hospital Regional Hans Dieter Schimidt. Joinville, Santa Catarina, SC
}

\section{*Correspondência}

Rua Jacob Eisenhuth, 400 -

apto 403

Bairro: Atiradores

CEP: 89203070

Joinville - Santa Catarina

Telefone: (47)30274817 /

(47) 99158559

moro_adriana@yahoo.com.br

\section{RESUMO}

Oвjetrvos. Determinar a prevalência e analisar o perfil dos portadores de lesão por pressão, enfocando fatores de risco, características clínicas e demográficas dos pacientes internados em hospital geral e estádio e localização das lesões no corpo. Métodos. Estudo transversal observacional não controlado. Foram observados diariamente os pacientes internados em hospital geral no período de abril a junho de 2005, a fim de identificar todos os casos de lesão por pressão. Os pacientes acometidos foram avaliados através de um questionário padronizado e a Escala de Braden foi aplicada para definir o risco de desenvolvimento das lesões.

Resultados. Do total de 690 pacientes internados durante o período referido, observou-se uma prevalência de 5,9\% de portadores de lesão por pressão, equivalente a 41 pacientes. Desses, 63,9\% eram idosos. A média do tempo de internação foi de 18 dias. Observou-se que o setor de clínica médica e a Unidade de Terapia Intensiva (UTI) apresentaram prevalência de 41,5\%. Quanto à localização das lesões, 73, $1 \%$ apresentavam-na em região sacral, e o grau mais evidenciado foi o estádio II, perfazendo um total de 58,5\%. De acordo com a Escala de Braden, a maioria (80,4\%) apresentava alto risco para 0 desenvolvimento das lesões, comparado com 9,7\% dos pacientes com risco moderado e 7,4\% com baixo risco.

Conclusão. Os pacientes acometidos apresentaram alto risco de desenvolver lesões por pressão. A prevalência dessas e o perfil clínico e demográfico dos pacientes acometidos estão de acordo com os dados encontrados na literatura.

Unitermos: Úlcera de decúbito. Prevalência. Epidemiologia.

\section{INTRODUÇÃO}

Úlceras por pressão são definidas pelo National Pressure Ulcer Advisory Panel (NPUAP) como "áreas localizadas de tecido necrótico que tendem a se desenvolver quando um tecido é comprimido entre uma proeminência óssea e uma superfície externa por tempo prolongado"!. Gradualmente, instala-se um processo isquêmico que resulta em uma lesão na pele, observada em quatro estádios de desenvolvimento, dependentes da manutenção dos fatores predisponentes ao seu surgimento ${ }^{2}$. Segundo - NPUAP, o método de classificação mais amplamente revisado, as úlceras por pressão classificam-se:

Estádio I - Lesão eritematosa não esbranquiçada em pele intacta. Descoloração, aumento de temperatura, edema ou endurecimento podem ser indicadores.

Estádio II - A superfície lesada encontra-se desunida da epiderme, derme ou ambas, apresentando-se de forma abrasiva, bolha ou despitelização rasa.

Estádio III - Perda tecidual acometendo área de tecido subcutâneo, que pode estender-se até a fáscia subjacente.

Estádio IV - Perda tecidual extensa e necrose de músculos, osso e/ou tendões subjacentes.

Embora consagrado pelo uso, o termo úlcera descreve um estádio mais avançado da lesão, ou seja, compreende a partir do estádio II. Dessa forma, os autores optaram por referirem-se a lesões por pressão. Com freqüência, encontram-se estudos apon- tando os principais fatores predisponentes para o desenvolvimento das lesões, descritos em dois grupos: fatores intrínsecos, inerentes à apresentação clínica do paciente, como idade, estado nutricional, perfusão tecidual e doenças associadas; fatores extrínsecos, relativos à exposição física do paciente, como fricção, cisalhamento, umidade e pressão, sendo essa, fator causal principal do desenvolvimento da lesão $0^{3-6}$.

Quanto às características epidemiológicas, diversos dados demonstram a magnitude desse acometimento em instituições de cuidados tanto primários quanto terciários ${ }^{6,7}$. Dessa forma, é descrita a prevalência dessas lesões em casas de atendimento terciário e hospitais gerais, como no estudo de Lahmann ${ }^{8}$, o qual encontrou uma prevalência de $\mid 1,7 \%$ entre os anos de $200 \mid$ e 2002, na Alemanha. Gunninberg', Chauhan ${ }^{10}$ e Woodbury 1 descrevem uma prevalência variando entre $5 \%$ e 23,9\%, possibilitando ter-se idéia do âmbito dessa doença. Diante disso, a prevalência de lesões por pressão nas instituições de atendimento representa maiores gastos para as mesmas, maior tempo de internação, piora na qualidade de vida e aumento da morbi-mortalidade desses pacientes 2-5, 8, 12 .

A avaliação do risco para seu desenvolvimento é realizado pela Escala de Braden, instrumento amplamente utilizado para esse fim $^{13}$. Estudos como o de Seongsook ${ }^{12}$, Bergstron ${ }^{13}$ e o de Blumel/4 descrevem a validade da escala para o estudo dessas lesões.

Dessa forma, é imprescindível que os profissionais da saúde atuem com a finalidade de prevenir o surgimento das lesões. Com 
um bom conhecimento da etiologia da doença, pressupõe-se que - manejo desses doentes seja otimizado ${ }^{15}$. Diante disso, este trabalho objetiva estabelecer o perfil dos portadores de lesões por pressão internados em hospital geral na cidade de Joinville - Santa Catarina. Contribuindo para o reconhecimento dos pacientes mais susceptíveis ao seu desenvolvimento, poderá se estabelecer manejo especial a esses pacientes, principalmente no que se refere à adoção de medidas profiláticas.

\section{Métodos}

O presente trabalho caracteriza-se como um estudo transversal, visto aferir os fatores causais e o desfecho num mesmo patamar temporal. Dessa forma, os sujeitos da pesquisa foram incluídos já apresentando o desfecho clínico - lesão por pressão - e os questionários aplicados, subseqüentemente descritos, foram analisados concomitantes. De acordo com os eixos de intervenção e controle, esse estudo é observacional não controlado.

A pesquisa foi realizada entre primeiro de abril a 30 de maio de 2005, no Hospital Hans Dieter Schmidt da cidade de Joinville-SC, que conta com 285 leitos distribuídos em diversas unidades de internação. Foram realizadas visitas diárias à instituição para a observação de todos os pacientes hospitalizados, independentemente do motivo da hospitalização, e posteriormente incluídos na pesquisa apenas os portadores de lesão por pressão desenvolvida dentro do hospital. Após determinados os sujeitos da pesquisa, os dados foram coletados através de um questionário contendo dados demográficos, dados clínicos gerais, como razão da internação, tempo de hospitalização, co-morbidades e dados referentes aos fatores de risco para desenvolvimento das lesões.

O risco para desenvolvimento de lesão por pressão foi determinado pela Escala de Braden, que é composta por seis subescalas que refletem a percepção sensorial do paciente, grau de umidade a qual a pele está exposta, atividade física, mobilidade, fricção e cisalhamento e estado nutricional do paciente. Todas são pontuadas de um a quatro, com exceção da fricção e do cisalhamento, que varia de um a três. Os escores totais variam de 6 a 23, sendo que os mais altos valores indicam baixo risco de formação, e os baixos escores indicam risco elevado ${ }^{13}$.

Após identificar os locais que apresentavam a lesão, foi estabelecida uma graduação conforme a classificação sugerida pelo NPUAP, baseada no local de maior acometimento.

A pesquisa foi aprovada pelo Comitê de Ética em Pesquisa da Universidade da Região de Joinville-SC e considerada uma pesquisa sem riscos para os participantes.

\section{Resultados}

Foram analisados 690 pacientes internados no período de primeiro de abril a 30 de maio, encontrando uma prevalência de $5,9 \%$ de portadores de lesão por pressão, correspondendo a 41 pacientes. Desses, $56 \%$ eram do sexo feminino e maioria era constituída por brancos $(75,6 \%)$. Com relação à idade, observouse predomínio de pacientes idosos (idade maior que 60 anos), equivalendo a $63,9 \%$.
Quanto aos setores visitados, apenas apresentaram pacientes portadores da lesão o setor de clinica médica $(41,5 \%)$, setor cirúrgico (I7\%) e a unidade de terapia intensiva (UTI) (4I,5\%); não foram encontrados pacientes acometidos no setor de pediatria nem de emergência.

A média do tempo de internação até o momento da pesquisa foi de 18 dias e quando estratificada a média por setor, os pacientes da ala clínica estavam internados havia 18 dias, os pacientes da UTI havia 12 dias e os do setor cirúrgico havia aproximadamente 23 dias. Quanto ao tempo médio de surgimento das lesões, a partir do momento da admissão, os pacientes do setor cirúrgico, clínico e da UTI desenvolveram a doença em aproximadamente 13,8 e 10 dias, respectivamente.

Entre as causas de hospitalização, houve predomínio de doenças infecciosas como pneumonia e infecção do trato urinário $(41,5 \%)$ e as demais doenças subdivididas como se segue: seis pacientes com problemas cardiovasculares (I4,6\%), seis pacientes $(14,6 \%)$ admitidos por conseqüência à doença neurológica, 10 pacientes decorrentes de intervenção cirúrgica $(24,4 \%)$ e dois pacientes com neoplasia $(4,9 \%)$.

Considerando que a incontinência urinária é um fator de risco, foram identificados 36 pacientes $(87,8 \%)$ com alguma alteração no sistema urinário, como uso de fraldas descartáveis ou sonda vesical de demora, contra 12,2\% sem alterações. Em relação aos antecedentes clínicos, oito pacientes (19,5\%) não apresentavam doença clínica associada, 10 pacientes $(24,4 \%)$ tinham apenas hipertensão arterial sistêmica, dois pacientes (4,9\%) apenas diabetes mellitus, II pacientes $(26,8 \%)$ apresentavam as duas doenças, 12,2\% apresentavam, além das duas morbidades, alguma outra não especificada aqui. Para aqueles não hipertensos e não diabéticos, portadores de outra doença clínica associada, obteve-se um valor de 12,2\% do total, sendo anemia a doença mais prevalente, encontrada em cinco pacientes, seguida de algum distúrbio respiratório.

No que diz respeito à localização, a maioria $(73,1 \%)$ apresentava o acometiomento em região sacral, seguido pelo calcâneo (30,3\%), acompanhado pela região de ísquio esquerdo. Sobre o total de portadores das lesões, 21,9\% apresentaram dois ou mais locais da doença. Esses dados são conferidos na Tabela I.

Conforme a escala de Braden, três pacientes (7,4\%) apresentavam grau baixo quanto ao risco de desenvolvimento das lesões por pressão e quatro pacientes com risco moderado (9,7\%). Trinta e quatro $(82,9 \%)$ apresentavam risco elevado, conforme mostrado na Tabela 2.

De acordo com o estádio de acometimento, encontraram-se: I5 pacientes (36,6\%) com estádio I, 24 (58,5\%) apresentando estádio II, dois $(4,9 \%)$ com grau III e nenhum paciente com grau IV, como segue-se na Tabela 3.

\section{Discussão}

As lesões por pressão representam uma das principais complicações que acometem pacientes críticos hospitalizados. São considerados pacientes críticos aqueles que têm condições clínicas graves ou necessidade de controles mais freqüentes e rigorosos, associados às terapias de maior complexidade ${ }^{15}$. 


\begin{tabular}{lc}
\hline \multicolumn{2}{c}{ Tabela I - Localizações mais freqüentes das lesões por pressão } \\
\hline Localização & $\frac{\mathbf{n}}{30}$ \\
Sacro & 2 \\
Ísquio esquerdo & 1 \\
Ísquio direito & 0 \\
Trocânter esquerdo & 0 \\
Trocânter direito & 9 \\
Calcâneo esquerdo & 8 \\
Calcâneo direito & 5 \\
Outros & \\
(occipital, orelha, maléolo direito) & \\
\hline
\end{tabular}

Tabela 2 - Estratificação do risco para o desenvolvimento de lesões por pressão

\begin{tabular}{|c|c|c|}
\hline Escala de Braden & $\mathrm{n}$ & $\%$ \\
\hline Alto risco & 34 & 82,9 \\
\hline Risco moderado & 4 & 9,7 \\
\hline Baixo risco & 3 & 7,4 \\
\hline Total & 41 & 100,0 \\
\hline
\end{tabular}

Tabela 3 - Estádios das lesões por pressão utilizando o National Pressure Ulcer Advisory Panel

\begin{tabular}{lcc}
\hline Estádio & $\mathbf{n}$ & $\frac{\%}{15}$ \\
\cline { 2 - 3 } Estádio I & $\mathbf{1 5}$ & 36,6 \\
Estádio II & 24 & 58,5 \\
Estádio III & 2 & 4,9 \\
Estádio IV & 0 & 0 \\
Total & 41 & 100,0 \\
\hline
\end{tabular}

Nesse contexto, é importante avaliar os fatores de risco para agravamento do quadro clínico do paciente, possibilitando que a equipe intervenha principalmente de forma profilática. Nesse estudo, procurou-se estabelecer as características epidemiológicas e alguns fatores potenciais para o acometimento em pacientes internados em hospital geral.

Os resultados apresentaram maior prevalência de lesões por pressão em pacientes idosos (63,9\%), havendo concordância com a literatura. Sabe-se que pacientes idosos estão sob maiores condições de risco para o agravamento do quadro inicial e, dessa forma, a probabilidade de desenvolvimento eleva-se dramaticamente com o aumento da idade $5,7,9,12,15-17$.

A cor da pele predominante foi a branca (75,6\%). Tal fato pode ser justificado pelo predomínio da população caucasiana em nosso meio. Além disso, é relatado na literatura que a pele negra tem maior resistência à agressão externa causada pela umidade e fricção, somado à dificuldade em identificar lesões de estádio I em indivíduos negros, o que pode ter contribuído para subestimar os casos encontrados nessa população ${ }^{15}$.

Foram analisadas todas as unidades de internação e de atendimento emergencial, obtendo igual prevalência de pacientes acome- tidos na clínica médica (4I,5\%) e UTI (41,5\%). A clínica cirúrgica apresentou $17 \%$ dos casos encontrados no hospital. Os pacientes em terapia intensiva geralmente apresentam alto risco para desenvolvimento, principalmente devido à diminuição da percepção sensorial causada por sedativos, analgésicos e relaxantes musculares, determinando menor reação à pressão excessiva. Segundo Bours et al, a incidência dessas lesões em pacientes internados em UTI pode variar de $1 \%$ a $43 \%{ }^{18}$.

Pesquisas demonstram que 0 tempo prolongado de internação está relacionado ao maior desenvolvimento de lesões por pressão. Lindgren et al encontraram que o tempo médio de hospitalização para pacientes que desenvolveram foi de 16 dias, contrapondo-se aos oito dias daqueles que não desenvolveram 6 . O presente estudo constatou que o tempo médio de internação foi de 18 dias, sendo que $68,3 \%$ dos pacientes desenvolveram a lesão em menos de 10 dias.

Considerando a causa da internação, observou-se um predomínio de doenças infecciosas (41,5\%), seguido de intervenções cirúrgicas $(24,4 \%)$. Sabe-se que pacientes submetidos a cirurgias de grande porte, nas quais a posição supina é exigida, estão predispostos ao acometimento, principalmente sacral e calcânea ${ }^{19,20}$. As doenças neurológicas representavam 14,6\% das hospitalizações, caracterizando pacientes com vários fatores de risco, como diminuição da atividade e da percepção sensorial. É relevante descrever a ausência de pacientes com paraplegia neste estudo, visto que essa afecção constitui-se fator predisponente a lesão por pressão.

No contexto das doenças preexistentes, houve prevalência da hipertensão arterial sistêmica $(24,4 \%)$ e diabetes mellitus (4,9\%). No diabetes ocorre um desequilíbrio entre o fornecimento e a demanda de insulina, podendo esses pacientes vir a ter complicações vasculares periféricas e diminuiç̧ão da sensibilidade; fatores de risco para a formação das lesões ${ }^{5,10}$.

Outro fator importante é a exposição da pele à umidade excessiva, tornando-a mais vulnerável à maceração e enfraquecimento de suas camadas superficiais ${ }^{3}$. Dos pacientes estudados, 87,8\% apresentavam alguma disfunção no sistema urinário. Entretanto, $70 \%$ estavam em uso de sonda vesical de demora, o que diminui a possibilidade da presença desse fator de risco.

Quanto ao local de acometimento, encontrou-se maior prevalência na região sacral, seguida dos calcâneos e ísquios. Diversos estudos evidenciam resultados semelhantes quanto à presença de lesões no sacro e calcâneos' $2,5,6,21,22$. Entretanto, há controvérsias quanto ao terceiro sítio de maior acometimento. Groeneveld et $a^{21}$ e Young apontam a orelha, enquanto que Lindgren ${ }^{6}$ relata o ísquio, confirmando os resultados encontrados no presente estudo.

Para avaliação do risco de desenvolvimento, optou-se pela escala de Braden, por ser o instrumento previamente validado e mais amplamente utilizado. Apresenta sensibilidade de $97 \%$ e especificidade de $26 \%$, valor preditivo positivo de $37 \%$ e valor preditivo negativo de $95 \%{ }^{12}$. A amostra estudada classificou $82,9 \%$ dos pacientes como alto risco, 9,7\% como risco moderado e 7,4\% como risco baixo. Esses resultados sugerem que essa escala 
deveria ser utilizada em todos os pacientes no momento da admissão, a fim de identificar aqueles com alto risco de desenvolver lesões por pressão e então provê-los com cuidados intensivos apropriados. Como cuidados profiláticos e paliativos para formação de lesões por pressão, nesta instituição, são utilizados colchões piramidais, mudança de decúbito, travesseiros entre as pernas para elevar os calcâneos, manutenção do lençol seco e esticado, hidratação da pele com óleo de girassol; quando já instalado o processo de formação da lesão utiliza-se placas de hidrocolóide.

Em relação ao estadiamento, encontrou-se maior prevalência de lesões grau II (58,5\%), contrapondo-se à maioria dos estudos que frequentemente identificam as pré-úlceras $5^{5,21,22}$. É possível que esse fato deva-se à dificuldade em distinguir entre a resposta fisiológica normal da pele à interface da superfície externa e a proeminência óssea, isto é, o eritema reativo, daquele não reativo, que já constitui a pré-lesão e poderá evoluir para estágios posteriores caso não se afaste o estímulo causal' ${ }^{15}$.

Ao considerar as características encontradas na população em estudo, bem como as revisões realizadas a respeito do tema, tornase pertinente a discussão dos fatores envolvidos no desenvolvimento dessas afecções como uma doença secundária passível de intervenção precoce.

Sabe-se que muitas das doenças secundárias podem ser prevenidas se abordadas precocemente, no entanto, no processo de saúde/doença na sociedade de um modo geral, isso ainda não está posto como princípio fundamental, uma vez que os investimentos na saúde ainda são muito mais afetos ao processo curativo. No que se refere à lesão por pressão, a atitude preventiva é essencial para melhorar a qualidade de vida ou de sobrevida de muitos pacientes que se encontram restritos ao leito ou à cadeira de rodas 3,5 .

Dessa forma, os profissionais de saúde devem estar preparados para atender aqueles que apresentam alto risco para o surgimento das lesões. Sabe-se que há diversos fatores que dificultam um melhor atendimento a esses pacientes, como a falta de uniformização do conhecimento em relação às medidas profiláticas. Além disso, o número reduzido de funcionários, somado à sobrecarga de trabalho, compromete a realização de mudança de decúbito a cada duas horas.

Por conseguinte, cabe aos profissionais de saúde identificar os pacientes de risco e elaborar propostas de prevenção, divulgandoas entre todos os envolvidos que possam, de alguma maneira, intervir para que seja modificada a história da doença.

\section{ConClusão}

A população estudada caracterizou-se por predomínio de pacientes brancos, portadores principalmente de lesão grau II, acometendo região sacral.

Além disso, foi observada uma amostra com risco elevado para desenvolver lesões por pressão. Dentre os fatores mais freqüentes, destacam-se a idade avançada, longo período de internação e imobilidade, sendo fundamental adotar medidas profiláticas adequadas, principalmente durante o período de internação.

\section{SUMMARY \\ ASSESSMENT OF PATIENTS WITH PRESSURE SORES ADMITTED IN A TERTIARY CARE CENTER}

BACKGROUND: To determine the prevalence and analyze the profile of patients with pressure sores, focusing on risk factors, the patients' clinical characteristics at a tertiary care center, as well as stage and location of the lesions on the body.

METHODS: This was a cross sectional not controlled observational study, all patients admitted from April to June of 2005 were observed daily to identify all cases of pressure sores. The affected patients were evaluated by a standard questionnaire and the Scale of Braden was applied to define the risk of developing ulcers.

RESULTS: Of the 690 patients admitted during the referred period, a prevalence of $5.9 \%$ of patients with lesions was observed, equivalent to 41 patients $63.9 \%$ of which were elderly and the average length of stay was 18 days. In the sample studied $41.5 \%$ of patients were found in the internal medicine section and the intensive care unit, ICU. The most common location for sores was the sacral area, corresponding to $73.1 \%$ of the patients, and stage II was the most frequent, observed in 58.5\% of those patients. According to the Braden scale, most patients, $80.4 \%$, had a high risk of developing pressure ulcers, compared to $9.7 \%$ of patients with moderate risk and $7.4 \%$ with low risk.

CONCLUSION: The affected patients were at high risk of developing pressure sores. Prevalence of these lesions and the clinical and demographic profile of the affected patients are in accordance with the data in literature. [Rev Assoc Med Bras 2007; 53(4): 300-4]

KEY WORDs: Pressure sores. Prevalence. Epidemiology.

\section{REFERÊNCIAS}

1. Pressure ulcer prevalence, cost and risk assessment: Consensus Development Conference statement. Decubitus. 1989;2:24-8.

2. Wong VK, Stotts NA. Physiology and prevention of heel ulcers: the state of science. J Wound Ostomy Continence Nurs. 2003;30:191-8.

3. Cooney LM Jr. Pressure sores and urinary incontinence. J Am Geriatr Soc. 1997;45:1278-9.

4. Carlijn V, et al. The Etiology of Pressure Ulcers: Skin Deep or Muscle Bound? Arch Phys Med Rehabil. 2003;84:616-9.

5. Young J, Nikoletti S, McCaul K, Twigg D, Morey P. Risk factors associated with pressure ulcer development at a major western Australian teaching hospital from 1998 to 2000: secondary data analysis. J Wound Ostomy Continence Nurs. 2002;29:234-4l.

6. Lindgren $M$, Unosson M, Fredrikson M, Ek A. Immobility: a major risk factor for development of pressure ulcers among adult hospitalezed patients: a prospective study. Scand J Caring Sci. 2004; 18:57-64.

7. Mathus-Vliegen EMH. Old age, malnutrition, and pressure sores: na illfated alliance. J Gerontol. 2004;59:335-60.

8. Lahmann NA, Halfens RJ, Dassen T. Prevalence of pressure ulcers in Germany. J Clin Nurs. 2005; |4:165-72.

9. Gunninberg L. Risk, prevalence and prevention of pressure ulcers in three Swedish healthcare settings. J Wound Care. 2004; 13:286-90.

1 0. Chauhan VS, Goel S, Kumar P, Srivastava S, Shukla VK. The prevalence of pressure ulcers in hospitalized patients in a university hospital in India. J Wound Care. 2005; |4:36-7. 
Moro A et AL.

II. Woodbury MG, Houghton PE. Prevalence of pressure ulcers in Canadian healthcare settings. Ostomy Wound Manage. 2004;50:22-38.

I 2. Seongsook J, Ihnsook J, Younghee L. Validity of pressure ulcer risk assessment scales; Cubbin and Jackson, Braden, and Douglas scale. Int J Nurs Stud. 2004;41:199-204.

13. Bergstron N, Braden B], Laguzza A, Holman V. The Braden Scale for predicting pressure score risk. Arch Intern Med. 1998;| 48:224I-3.

I 4. Blumel JE, Tirado K, Schiele C, Schonffeldt G, Sarrá S. Validez de la escala de Braden para predecir úlceras por presión en población femenina. Rev Med Chile. 2004; I32:595-600.

15. Blanes L, Duarte IS, Calil JA, Ferreira LM. Avaliação clínica e epidemiológica das úlceras por pressão em pacientes internados no Hospital São Paulo. Rev Assoc Med Bras. 2004;50:182-7.

16. Jorge SA, Dantas, SRPE. Úlceras de pressão. In: Paranhos WY. Abordagem multidisciplinar do tratamento de feridas. São Paulo: Ed Atheneu; 2003. p.287-98.

17. Tannen A, Dassen T, Bours G, Halfens R. A comparison of pressure ulcer prevalence: concerted data collection in the Netherlands and Germany. Int I Nurs Stud. 2004:41:607-12.

18. Bours GJJ, De Laat E, Halfens RJG, Lubbers M. Prevalence, risk factors and prevention of pressure ulcers in Dutch intensive care units. Intensive Care Med. 2001;27:1599-605.
19. Theaker C, Mannan M, Ives N, Soni N. Risk factors for pressure sores in the critically ill. Anaesthesia. 2000;55:221-4.

20. Nixon J, Brown J, McElvenny D, Mason S, Bond S. Prognostic factors associated with pressure sore development in the immediate postoperative period. Int J Nurs Stud. 2000;37:279-89.

21. Groeneveld A et al. The prevalence os pressure ulcers in a tertiary care pediatric and adult hospital. I Wound Ostomy Continence Nurs. 2004;31: 108-20.

22. Whittington K, Patrick M, Roberts JL. A national study of pressure ulcer prevalence and incidence in acute care hospitals. J Wound Ostomy Continence Nurs. 2000;27:209-I5.

Artigo recebido: 09/08/06

Aceito para publicação: 21/05/07 